\title{
Artículo Original \\ Experiencia en Cirugía de Miocardiopatía Hipertrófica Obstructiva en un Centro de Referencia Nacional
}

Víctor Robles, ${ }^{1 *}$ Yemmy Pérez, ${ }^{1}$ Josías Ríos ${ }^{1}$

Recibido 10 de marzo de 2020 Aceptado 8 de abril de 2020

Afiliaciones de los Autores:

1 Servicio de Cirugía Cardiovascular Adulto - Instituto Nacional Cardiovascular INCOR. Lima, Perú.

\section{${ }^{*}$ Correspondencia:}

Instituto Nacional Cardiovascular INCOR. Jr. Coronel Zegarra 417.

Jesús María. Lima 11. Perú.

Telef. 01-4111560, anexo 5931.

Correo:

victoroblesccv@gmail.com

\section{Conflicto de interés:}

No se reporta conflicto de interés.

\section{Responsabilidades éticas:}

Protección de personas y animales. Los autores declaran que para esta investigación no se ha realizado experimentos en seres humanos $n$ en animales.

Confidencialidad de los datos: Los autores declaran que han seguido los protocolos de su centro de trabajo sobre la publicación de datos de pacientes.

Derecho a la privacidad y consentimiento informado:

Los autores declaran que en este artículo no aparecen datos de pacientes.

Financiación:

Ninguna.

\section{RESUMEN}

Objetivos: Identificar las características clínicas, analizar los resultados y mostrar la eficacia del tratamiento quirúrgico de la miocardiopatía hipertrófica obstructiva (MHO), en un hospital de referencia nacional

Material y métodos: Se realizó un estudio descriptivo, retrospectivo, tipo serie de casos, de pacientes operados en el Instituto Nacional Cardiovascular con el diagnóstico de MHO, entre diciembre del 2016 y enero del 2019. Se analizó la evolución posoperatoria de la sintomatología, clase funcional (CF), gradiente del tracto de salida del ventrículo izquierdo (GTSVI) y de la insuficiencia mitral (IM).

Resultados: Se evaluaron 13 casos con MHO sometidos a miectomía septal extendida. Un $31 \%$ fueron mujeres y la edad media fue de 39.6 años. Antes del tratamiento quirúrgico $85 \%$ se encontraba en CF III-IV, $85 \%$ de pacientes presentaba IM severa, el grosor medio del septum interventricular era de 27 $\mathrm{mm}$ (rango de 19 a $39 \mathrm{~mm}$ ) y el GTSVI medio, de $111 \mathrm{mmHg}$ (rango de 60 a $150 \mathrm{mmHg}$ ). Luego del tratamiento quirúrgico se observó mejoría de la CF (69\% en CF I)y del grado de IM ( $92 \%$ con IM nula o mínima), y reducción de GTSVI media a $16 \mathrm{mmHg}$ (rango de 6 a $35 \mathrm{mmHg}$ ). En 7 pacientes (54\%) se realizó cirugía simultánea de la válvula mitral.

Conclusiones: Nuestra experiencia en cirugía de MHO es adecuada. El tratamiento simultáneo de los componentes miocárdicos y valvular permite reducir el GTSVI y corregir la IM.

Palabras Clave: miocardiopatía hipertrófica • cirugía • miectomía septal

\section{ABSTRACT}

\section{Experience in Obstructive Hyperthrophic Myocardiopathy} Surgery at a National Reference Center

Objectives: To identify the clinical characteristics, analyze the results and show the efficacy of surgical treatment of hypertrophic obstructive cardiomyopathy $(\mathrm{HOC})$, in a national reference institute.

Methods: A descriptive, retrospective, case series of patients with the diagnosis of $\mathrm{HOC}$ operated at the National Cardiovascular Institute, was performed between December 2016 and January 2019. We analyzed the postoperative evolution of symptomatology, functional class (FC), left ventricular outflow tract gradient (LVOTG) and mitral regurgitation (MR).

Results: Thirteen cases with HOC undergoing extended septal myectomy were evaluated. $31 \%$ were women and the average age was 39.6 years. Before surgical treatment, $85 \%$ were in functional class (FC) III-IV, 85\% of patients had severe MR, the mean septum thickness was $27 \mathrm{~mm}$ (range from 19 to 39 $\mathrm{mm}$ ), and mean LVOTG was $111 \mathrm{mmHg}$ (range from 60 to $150 \mathrm{mmHg}$ ). After surgical treatment we found improvement of the functional class ( $69 \%$ in $\mathrm{FCI}$ ) and the degree of MR ( $92 \%$ with zero or minimal), and reduction of mean LVOTG to $16 \mathrm{mmHg}$ (range from 6 to $35 \mathrm{mmHg}$ ). Simultaneous surgery of the mitral valve was performed in 7 patients (54\%).

Conclusions: Our experience in HOC surgery is good. The treatment of both myocardial and valve components allows reducing LVOTG and correcting MR.

Keywords: hypertrophic obstructive cardiomyopathy • surgery • septal myectomy 
$\mathrm{E}$

studios epidemiológicos basados en estudios ecocardiográficos muestran una prevalencia de la miocardiopatía hipertrófica obstructiva $(\mathrm{MHO})$ de 1 caso por 500 personas en la población general, con ligero predominio del sexo masculino. ${ }^{1,2}$ Etiológicamente, en un $60 \%$ de los adolescentes y adultos con MHO la enfermedad tiene un rasgo autosómico dominante, causado por mutaciones en más de 11 genes que codifican los componentes proteicos del sarcómero. ${ }^{3}$

La fisiopatología de la MHO, está caracterizada por una obstrucción dinámica, de intensidad variable, normalmente localizada en posición subvalvular aórtica, entre el septum interventricular hipertrofiado y la válvula mitral, asociada con frecuencia a un movimiento sistólico anterior (MAS) de la vávula mitral que produce diversos grados de insuficiencia mitral (IM). ${ }^{1}$

El diagnóstico de la MHO se basa en la detección de un aumento del grosor de la pared del ventrículo izquierdo (VI) mediante cualquier modalidad de imagen (ecocardiografía, resonancia magnética, tomografía computarizada). En el adulto se define por un grosor de la pared $\geq$ de $15 \mathrm{mmm}$ en uno o más segmentos miocárdicos del $\mathrm{Vl}$, que no pueda explicarse únicamente por condiciones de carga. ${ }^{4}$ La obstrucción del tracto de salida del ventrículo izquierdo (OTSVI) se define como un gradiente doppler instantáneo máximo del tracto de salida del ventrículo izquierdo $\geq 30 \mathrm{mmHg}$, pero el umbral a considerar para el tratamiento invasivo es de $\geq 50$ $\mathrm{mmHg} .{ }^{4}$ En la evolución clínica de la $\mathrm{MHO}$, la mayoría no presentan síntomas clínicos y/o eventos adversos relevantes, no requieren tratamiento y cursan con una esperanza de vida normal. Un 5\% de los pacientes evolucionan con síntomas persistentes y son candidatos a una terapia invasiva, ya sea con el desfibrilador automático implantable (DAl), ablación miocárdica septal transluminal percutánea o cirugía. ${ }^{5}$

El tratamiento invasivo para reducir la OTSVI se debe considerar en pacientes con un gradiente $\geq 50 \mathrm{mmHg}$, síntomas de moderados a graves (clase funcional NYHA III - IV), O síncope de esfuerzo recurrente a pesar de recibir tratamiento farmacológico a dosis máximas toleradas. ${ }^{6}$ Por la complejidad del sustrato anatómico que produce la obstrucción en la MHO se describe diferentes estrategias quirúrgicas que se han reportado en los últimos años. ${ }^{78}$ El tratamiento quirúrgico más usual para tratar la OTSVI es la miectomía ventricular (procedimiento de Morrow). ${ }^{9}$ Un grupo de pacientes requiere cirugía mitral concomitante como la sustitución de la válvula mitral, realineación, escisión parcial o movilización de los músculos papilares, plicatura o extensión de la valva anterior. ${ }^{10}$ Otra opción quirúrgica es la miectomía septal mínimamente invasiva transmitral con reparación y/o reemplazo de la válvula mitral. ${ }^{11}$
El objetivo de este trabajo fue analizar los resultados del tratamiento quirúrgico en pacientes con $\mathrm{MHO}$ que recibieron terapia farmacológica máxima sin mejoría clínica, en el Instituto Nacional Cardiovascular INCOR del Perú.

\section{Material y Método}

Se realizó un estudio descriptivo, retrospectivo, tipo serie de casos, de pacientes con $\mathrm{MHO}$, intervenidos quirúrgicamente en el Instituto Nacional Cardiovascular - INCOR EsSalud del Perú entre diciembre del 2016 a enero del 2019. La recolección de datos se realizó mediante la revisión de las historias clínicas. Se utilizó para el análisis y elaboración de resultados las siguientes variables: edad, sexo, antecedentes familiares, estudios diagnósticos realizados en el preoperatorio, signos y síntomas preoperatorios y postoperatorios, clase funcional preoperatorio y postoperatorio, grado de insuficiencia mitral (IM) en el preoperatorio y postoperatorio, fracción de eyección del ventrículo izquierdo (FEVI) preoperatorio, grosor del septum interventricular pre y postoperatoria, gradiente del tracto de salida del ventrículo izquierdo (GTSVI) pre y postoperatorio, técnica quirúrgica realizada, cirugía de la válvula mitral, uso de desfibrilador automático implantable (DAl) y estancia hospitalaria. Las variables numéricas se expresaron en media y desviación estándar, mediana y rango intercuartil y las categóricas en porcentajes. Se analizaron las diferencias entre grupos con la prueba de T de Student para muestras independientes o con la prueba en $\mathrm{U}$ de MannWhitney para variables continuas y la prueba de chi cuadrado para variables categóricas. Se consideró estadísticamente significativo el valor de $p<0.05$. Los datos fueron sometidos al análisis en el programa estadístico STATA 15.

\section{Resultados}

Entre diciembre del 2016 y enero del 2019 se intervinieron quirúrgicamente 13 pacientes con el diagnóstico de MHO en el Instituto Nacional Cardiovascular INCOR - EsSalud. Once pacientes (85\%) presentaban antecedentes familiares de MHO. La edad de los pacientes fluctuó entre los 14 años y los 67 años, y la mayoría de ellos fueron varones (69\%). Todos los pacientes tenían terapia farmacológica máxima sin mejoría de la sintomatología y 9 pacientes (69\%) eran portadores de DAI. (Tabla 1)

El estudio ecocardiográfico se realizó en el 100\% de los pacientes y el cateterismo cardíaco en $46 \%$. Los síntomas más frecuentes de presentación fueron disnea, angina y síncope. El grado de IM fue evaluado en el preoperatorio, intraoperatorio y postoperatorio por medio de ecografía transtorácica y transesofágica. En 12 pacientes se realizó la miectomía septal extendida según la técnica clásica (Morrow) 
y en 1 paciente se realizó miectomía septal transmitral por mini toracotomía anterolateral derecha videoasistida, cuyos resultados quirúrgicos fueron similares a la técnica clásica.

\section{Procedimiento quirúrgico}

La cirugía se realizó, en todos los casos, con control de ecocardiografía transesofágica. En 12 pacientes se realizó una esternotomía media, canulación en aorta ascendente y de ambas cavas, heparinización y entrada a circulación extracorpórea, llevando al paciente a una temperatura de $32^{\circ} \mathrm{C}$. La parada cardíaca con protección miocárdica se realizó usando cardioplejia sanguínea. Se realizó una aortotomía oblicua dirigida hacia el velo aórtico no coronariano para acceder al septum interventricular. Se separó cuidadosamente los velos aórticos y con un bisturí número 15 se practicó una resección de un fragmento del septum interventricular por medio de dos incisiones paralelas, la primera debajo del nadir del velo coronariano derecho y la segunda (en contra de las agujas del reloj) debajo de la comisura del velo coronariano derecho e izquierdo, respetando $5 \mathrm{~mm}$ debajo del anillo valvular aórtico. La resección se prolongó distalmente hasta la base de la implantación de los músculos papilares (miectomía septal extendida). (Tabla 2)

En 6 pacientes se realizó miectomía aislada (46\%) y en 7 pacientes (54\%) se realizó cirugía de válvula mitral concomitante (3 valvuloplastias y 4 implantes de prótesis valvular) con abordaje por atrio izquierdo. En el único caso de miectomía septal transmitral mínimamente invasiva, se hizo con canulación arterial y venosa vía femoral, y el abordaje fue por una minitoracotomía anterolateral derecha videoasistida. Al ter ${ }^{-}$ minar la circulación extracorpórea se realizó en todos los casos el control ecocardiográfico transesofágico para comprobar la reducción del GTSVI y la corrección de la IM.

\section{Desenlaces}

El 85\% de los pacientes en el preoperatorio se encontraban en CF III-IV y luego del tratamiento quirúrgico, el 100\% de los pacientes sobrevivientes pasaron a CF I-II. El gradiente medio del tracto de salida del VI disminuyó de $111.4 \mathrm{mmHg}$ (preoperatorio) a $15.7 \mathrm{mmHg}$ (postoperatorio). En cuanto a la IM, el $85 \%$ de los pacientes presentaba IM severa y en el postoperatorio el 92\% tuvo IM leve o nula. (Tabla 3)

Se encontraron dos eventos intrahospitalarios post cirugía: un paciente portador de DAI falleció al decimosegundo día postoperatorio por una arritmia sin respuesta al tratamiento. En otro paciente el control ecocardiográfico al primer día postoperatorio encontró IM severa a pesar de haberse realizado la valvuloplastia mitral por lo que se procedió a una segunda intervención para reemplazo valvular mitral sin complicaciones.
Tabla 1. Características basales

\begin{tabular}{lrl}
\hline Edad & 39.6 & $( \pm 17.4)$ \\
Varones & 9 & $(69)$ \\
Antecedente familiar de MHO & 11 & $(85)$ \\
Disnea & 13 & $(100)$ \\
Angina & 7 & $(54)$ \\
Síncope & 4 & $(31)$ \\
Portador de DAl & 9 & $(69)$ \\
\hline
\end{tabular}

Se reporta medias (desviación estándar) y frecuencias (porcentaje) para variables cuantitativas y categóricas, respectivamente. MHO: miocardiopatía hipertrófica obstructiva; DAl: defibrilador automático implantable.

\section{Tabla 2. Características del intraoperatorio}

\begin{tabular}{lrl}
\hline $\begin{array}{l}\text { Tiempo de pinzamiento de aorta } \\
\text { (minutos) }\end{array}$ & 82.6 & $( \pm 36.5)$ \\
$\begin{array}{l}\text { Tiempo de circulación extracorpórea } \\
\text { (minutos) }\end{array}$ & 138.2 & $( \pm 60)$ \\
$\begin{array}{l}\text { Plastía de la válvula mitral } \\
\text { (pacientes) }\end{array}$ & 3 & $(23.1)$ \\
$\begin{array}{l}\text { Reemplazo de la válvula mitral } \\
\text { (pacientes) }\end{array}$ & 4 & $(30.8)$ \\
$\begin{array}{l}\text { Tiempo en cuidado intensivo } \\
\text { (días) }\end{array}$ & 2.9 & $( \pm 1.1)$ \\
\hline
\end{tabular}

Se reporta medias (desviación estándar) y frecuencias (porcentaje) para variables cuantitativas y categóricas, respectivamente.

Tabla 3. Características ecográficas pre y postoperatorias

\begin{tabular}{lrllll}
\hline & \multicolumn{2}{c}{$\begin{array}{c}\text { Pre } \\
\text { Operatorio }\end{array}$} & \multicolumn{3}{c}{$\begin{array}{c}\text { Post } \\
\text { Operatorio }\end{array}$} \\
\hline Septum basal & 27.1 & $( \pm 6.1)$ & 19.3 & $( \pm 8.3)$ \\
Gradiente en TSVI & 111.4 & $( \pm 35.9)$ & 15.7 & $( \pm 9.8)$ \\
$\begin{array}{l}\text { Insuficiencia mitral } \\
\text { moderada o severa }\end{array}$ & 13 & $(100)$ & 1 & $(8.0)$ \\
\hline
\end{tabular}

Se reporta medias (desviación estándar) y frecuencias (porcentaje) para variables cuantitativas y categóricas, respectivamente. TSVI: Tracto de salida de ventrículo izquierdo

\section{Discusión}

La elección del tratamiento para la MHO es diversa y la historia natural, impredecible. Diversos estudios retrospectivos han estimado una mortalidad anual aproximada menor del $1 \%^{2,12}$ y muchas de las muertes son súbitas, presumiblemente causadas por arritmias ventriculares. ${ }^{1}$ Otras complicaciones que pueden ocurrir incluyen fibrilación auricular, endocarditis infecciosa y estadios finales de insuficiencia 
cardíaca. ${ }^{13}$ Los betabloqueadores y calcioantagonistas pueden dar mejoría sintomática a muchos pacientes con $\mathrm{MHO}$, especialmente en aquellos sin obstrucción u obstrucción latente, pero hay considerables variaciones en la respuesta individual a las drogas. ${ }^{14}$

El manejo depende de la sintomatología del paciente. En los sintomáticos se debe usar betabloqueadores y calcioantagonistas, y como última opción queda el manejo inva sivo que se divide en dos modalidades: ablación con alcohol y la miectomía quirúrgica que disminuye el grosor del septum, corrige la OTSVI y la IM. En un 5\% de los pacientes la sintomatología es refractaria al tratamiento farmacológico y se debe considerar terapia invasiva. ${ }^{6,15}$

Para los pacientes con MHO y OTSVI, el tratamiento quirúrgico da mejoría sustancial de los síntomas y puede mejorar el pronóstico. ${ }^{16,17}$ La miectomía septal transaórtica como procedimiento estándar fue desarrollado por Morrow et $a l^{9}{ }^{9}$ es excelente en liberar la OTSVI y es altamente reproducible. Otras alternativas quirúrgicas como la miectomía septal mínimamente invasiva se proponen seguras y requieren experiencia e instrumental especializado. ${ }^{11,18}$

La miectomía septal extendida incrementa el diámetro del tracto de salida del ventrículo izquierdo con la disminución del gradiente y la velocidad de flujo, y corrige la IM funcional. Sin embargo, el tratamiento debe considerarse no solo para el septum hipertrófico, sino también para la válvula mitral y el aparato subvalvular pues la presencia de alteraciones anatómicas de la válvula mitral en pacientes con MHO está bien documentada. ${ }^{19,20}$ Los velos mitrales son habitualmente más grandes de lo normal y los músculos papilares adoptan una posición más anterior dentro de la cavidad ventricular. En ese sentido, la plicatura transversal, ${ }^{21}$ y la movilización y escisión parcial de los papilares ${ }^{22}$ se describen como técnicas quirúrgicas para el manejo de patología mitral. Estas técnicas deben limitarse a aquellos con MAS, IM severa y velos grandes y redundantes.
El reemplazo valvular mitral, posiblemente el tratamiento más radical de la $\mathrm{MHO}$ para conseguir una mayor reducción de la GTSVI y corregir la IM, debe realizarse resecando toda la válvula y el aparato subvalvular, incluidos los músculos papilares. ${ }^{23}$ Si desaparece el velo anterior no existe posibilidad de MAS. Sin embargo, esta debe ser una alternativa en casos seleccionados, debido a las complicaciones propias de las prótesis. ${ }^{24}$

En nuestra experiencia, la cirugía logró una reducción significativa del GTSVI, de la IM y una mejoría sintomática. Las cifras del GTSVI mostradas se encuentran dentro de los límites de otros estudios internacionales (que oscilan entre 4.5 y 16 $\mathrm{mmHg}) \cdot{ }^{22,25}$ No se ha tenido ningún caso de comunicación interventricular ni insuficiencia aórtica iatrogénica, debido al respeto de los límites quirúrgicos ya descritos. Las cifras de mortalidad hospitalaria de la miectomía en centros de gran experiencia es menor al $2 \%$, el riesgo puede ser mayor en paciente ancianos y en aquellos con comorbilidades existentes. $^{26,27}$

La mayor limitación del presente estudio es el número pequeño de pacientes sometidos a cirugía y las dificultades en el seguimiento a largo plazo por ser nuestro instituto un centro de referencia nacional y debido a que el paciente postoperado, una vez resuelta la patología quirúrgica, retorna a su hospital de origen.

\section{Conclusión}

La miectomía septal extendida en pacientes con $\mathrm{MHO}$, corrige la obstrucción del tracto de salida del VI, la IM y se observa una mejoría en la capacidad funcional. EI tratamiento debe abarcar el componente miocárdico y valvular. Los resultados obtenidos en el presente estudio muestran que en nuestro instituto el tratamiento quirúrgico de la $\mathrm{MHO}$ es adecuado y se encuentra dentro de los parámetros internacionales.

\section{Referencias Bibliográficas}

1. Maron BJ. Clinical Course and Management of Hypertrophic Cardiomyopathy. N Engl J Med 2018;379:655-68.

2. Gersh BJ, Maron BJ, Bonow RO, et al. 2011 ACCF/AHA guideline for the diagnosis and treatment of hypertrophic cardiomyopathy: executive summary: a report of the American College of Cardiology Foundation/American Heart Association Task Force on Practice Guidelines. Circulation 2011;124:2761-96.

3. Alcalai R, Seidman JG, Seidman CE. Genetic basis of hypertrophic cardiomyopathy: From bench to the clinics. J Cardiovasc Electrophysiol. 2008;19:104-10.

4. Elliott PM, Anastasakis A, Borger MA, et al. 2014 ESC Guidelines on diagnosis and management of hypertrophic cardiomyopathy.
The Task Force for the Diagnosis and Management of Hypertrophic Cardiomyopathy of the European Society of Cardiology (ESC). European Heart Journal 2014;35:2733-79.

5. Spirito P, Seidman CF, McKenna WJ, et al. The management of hypertrophic cardiomiopathy. N Engl J Med 1997;336:775-85.

6. Maron BJ, Mathenge R, Casey SA, et al. Clinical profile of hypertrophic cardiomyopathy identified de novo in rural communities. J Am Coll Cardiol. 1999;33:1590-5.

7. Jahangiri M, Nicholson IA, Del Nido PJ, et al. Surgical Management of complex and tunel-like subaortic stenosis. Eur J Cardiothorac Surg. 2000;17:637-42. 
8. Candela-Navarro G, Aguilar-Jiménez JM, Valencia-Díaz YF, et al. Tratamiento quirúrgico de la miocardiopatía hipertrófica obstructiva mediante la técnica de Konno modificada. Cir Cardiov. 2013;20(1):42-5.

9. Morrow AG, Brockenbrough EC. Surgical Treatment of Idiopathic Subaortic Stenosis. Technic and Hemodynamic Results of Subaortic Ventriculomyotomy. Annals of Surgery 1961;154(2): 181-9.

10. Messmer BJ. Extended Myectomy for Hypertrophic Obstructive Cardiomyopathy. Ann Thorac Surg 1994;58:575-7.

11. Gilmanov DS, Bevilacqua $S$, Solinas $M$, et al. Minimally Invasive Septal Myectomy for the Treatment of Hypertrophic Obstructive Cardiomyopathy and Intrinsic Mitral Valve Disease. Innovations 2015;10:106-13.

12. Maron BJ. Hypertrophic cardiomyopathy: a systematic review. JAMA 2002;287:1308-20.

13. Maron BJ, Bonow RO, Cannon RO III, et al. Hypertrophic cardiomyopathy: interrelations of clinical manifestations, pathophysiology, and therapy. N Engl J Med 1987;316:844-52.

14. Rowin EJ, Maron MS, Chan RH, et al. Interaction of adverse disease-related pathways in hypertrophic cardiomyopathy. Am J Cardiol 2017; 120: 2256-64.

15. Vriesendorp PA, Liebregts $M$, Steggerda $R C$, et al. Long-term outcomes after medical and invasive treatment in patients with hypertrophic cardiomyopathy. JACC Heart Fail 2014; 2: 630-6.

16. Dearani JA, Ommen SR, Gersh BJ, et al. Surgery insight: Septal myectomy for obstructive hypertrophic cardiomyopathy - the Mayo Clinic experience. Nat Clin Pract Cardiovasc Med. 2007:4:503-11.

17. Castedo E, Cabo RA, Núñez l, et al. Tratamiento quirúrgico de la miocardiopatía hipertrófica obstructiva. Rev Esp Cardiol 2004;57(8):751-6.

18. Matsuda H. Transatrial and transmitral myectomy for hypertrophic obstructive cardiomyopathy of the left ventricle. Oper Tech Thorac Cardiovasc Surg. 2004;9:304-9.
19. Maron MS, Maron BJ. Clinical impact of contemporary cardiovascular magnetic resonance imaging in hypertrophic cardiomyopathy. Circulation 2015;132: 292-8.

20. Sherrid MV, Balaram S, Kim B, et al. The mitral valve in obstructive hypertrophic cardiomyopathy: a test in context. J Am Coll Cardiol 2016; 67:1846-58

21. Sherrid MV, Chaudhry FA, Swistel DG. Obstructive hypertrophic cardiomyopathy: echocardiography, pathophysiology, and the continuing evolution of surgery for obstruction. Ann Thorac Surg 2003;75:620-32.

22. Schoendube FA, Klues HG, Reith S, et al. Long-term clinical and echocardiographic follow-up after surgical correction of hypertrophic obstructive cardiomyopathy with extended myectomy and reconstruction of the subvalvular mitral apparatus. Circulation 1995;92(Suppl II):122-7. .

23. Cooley DA, Leachman RD, Wukasch DC. Mitral valve replacement for idiopathic hypertrophic cardiomyopathy. J Cardiovasc Surg 1976;17:380-7.

24. Vesey JM, Otto CM. Complications of prosthetic heart valves. Curr Cardiol Rep. 2004;6(2):106-11.

25. Schulte HD, Borisov K, Gams E, Gramsch-Zabel H, Losse B, Schwartzkopff B. Management of symptomatic hypertrophic obstructive cardiomyopathy-long term results after surgical therapy. Thorac Cardiovasc Surg 1999;47:213-8.

26. Maron BJ, Dearani JA, Ommen SR, et al. Low operative mortality achieved with surgical septal myectomy: role of dedicated hypertrophic cardiomyopathy centers in the management of dynamic subaortic obstruction. J Am Coll Cardiol. 2015;66:1307-08.

27. Desai MY, Bhonsale A, Smedira NG, et al. Predictors of Long-Term Outcomes in Symptomatic Hypertrophic Obstructive Cardiomyopathy Patients Undergoing Surgical Relief of Left Ventricular Outflow Tract Obstruction. Circulation. 2013;128:209-16. 